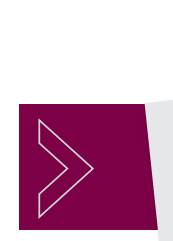

- Nausea

- Dizziness

\section{Courtney Lynn \\ Dominguez, MD;}

Jonathan Armstrong, MD; Adrian Billings, MD, PhD, FAAFP

Department of Internal

Medicine, Duke University Medical Center, Durham,

NC (Dr. Dominguez); Family Medicine with Obstetrics,

Sea Mar Community Health Center, Vancouver, WA (Dr. Armstrong); Department of Family and Community Medicine, Texas Tech University Health Sciences CenterPermian Basin, Odessa (Dr. Billings)

ت courtney.dominguez@ duke.edu

The authors reported no potential conflict of interest relevant to this article.

doi: 10.12788/jfp.0204

\title{
THE DIAGNOSIS
}

An increase in heart rate of more than 30 beats/min when the patient went from a sitting to a standing position pointed to a diagnosis of postural orthostatic tachycardia syndrome (POTS). This prompted us to stop the sertraline.

\section{DISCUSSION}

POTS is a type of intolerance to orthostasis related to a significant increase in pulse without resulting hypotension upon standing. Other symptoms that accompany this change in position include dizziness, lightheadedness, blurry vision, and fatigue. Syncope occurs in about $40 \%$ of patients with POTS, which may be more frequent than for patients with orthostatic hypotension. ${ }^{1}$

The overall prevalence of POTS is $0.2 \%$ to $1 \%$; however, it is generally seen in a 5:1 female-to-male ratio. ${ }^{2,3}$ POTS is often idiopathic. That said, it can also be caused by medi- 


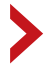

Case reports have shown an association between SSRIS and syncope. SSRIs have also been tied to increased heart rate variability. cation adverse effects, hypovolemia, and stressors, including vaccinations, viral infections, trauma, and emotional triggers. On physical exam, this patient did not appear to be hypovolemic, and she reported normal oral intake prior to this visit. Since the patient had started taking sertraline about 10 days prior to her syncopal episodes, we suspected POTS secondary to sertraline use was the likely etiology in this otherwise healthy young woman.

\section{Syncope could indicate a larger cardiovascular problem}

The differential diagnosis of dizziness with loss of consciousness includes anemia, vasovagal syncope, orthostatic hypotension, dehydration, electrolyte imbalance, arrhythmia, prolonged QT syndrome, cardiac valve or structure abnormality, and seizure. Most of these differentials can be ruled out from basic laboratory tests or cardiac imaging. In POTS, the diagnostic work-up is essentially normal compared to other causes of syncope. Orthostatic hypotension, for example, is similar; however, there is an additional change in the arterial blood pressure.

\section{Unintended adverse effects}

Selective serotonin reuptake inhibitors (SSRIs), such as sertraline, are known to have fewer cardiovascular adverse effects compared to older antidepressants such as tricyclic antidepressants and monoamine oxidase inhibitors. ${ }^{4}$ However, case reports have shown an association between SSRIs and syncope. ${ }^{4-6}$ SSRIs have also been tied to increased heart rate variability. ${ }^{7}$

I Nearly 2 weeks after stopping sertraline, our patient presented to clinic and was given a diagnosis of streptococcal pharyngitis. She said she'd had no additional syncopal episodes. Twenty days after sertraline cessation, the patient returned for follow-up. Her blood pressure and heart rate were as follows: supine, 112/68 mm $\mathrm{Hg}$ and 61 beats/min; seated, 113/74 $\mathrm{mm} \mathrm{Hg}$ and 87 beats/min; and standing, 108/74 $\mathrm{mm} \mathrm{Hg}$ and 78 beats $/ \mathrm{min}$.

Thus, after cessation of sertraline, her orthostatic heart rate changes were smaller than when she was first examined. Her vital signs showed an increase in pulse of 26 beats/min between lying and sitting, without any reports of nausea. She had no further complaints of dizziness or syncopal episodes.

\section{THE TAKEAWAY}

We don't always know how a patient will respond to a newly prescribed medication or lifestyle change. A proper review of a patient's history and medication use is a pivotal first step in making any diagnosis.

JFP

CORRESPONDENCE

Courtney Lynn Dominguez, MD, 4220 North Roxboro Street, Durham, NC 27704; courtney.dominguez@duke.edu

\section{REFERENCES}

1. Ojha A, McNeeley K, Heller E, et al. Orthostatic syndromes differ in syncope frequency. Am J Med. 2010;123:245-249. doi: 10.1016/j.amjmed.2009.09.018

2. Arnold AC, Ng J, Raj SR. Postural tachycardia syndromediagnosis, physiology, and prognosis. Auton Neurosci. 2018;215: 3-11. doi: 10.1016/j.autneu.2018.02.005

3. Fedorowski A. Postural orthostatic tachycardia syndrome: clinical presentation, aetiology and management. J Intern Med. 2018;285:352-366. doi:10.1111/joim.12852

4. Pacher P, Ungvari Z, Kecskemeti V, et al. Review of cardiovascular effects of fluoxetine, a selective serotonin reuptake inhibitor, compared to tricyclic antidepressants. Curr Med Chem. 1998;5:381-390.

5. Feder R. Bradycardia and syncope induced by fluoxetine. J Clin Psychiatry. 1991;52:139.

6. Ellison JM, Milofsky JE, Ely E. Fluoxetine-induced bradycardia and syncope in two patients. J Clin Psychiatry. 1990;51: 385-386.

7. Tucker P, Adamson P, Miranda R Jr, et al. Paroxetine increases heart rate variability in panic disorder. $J$ Clin Psychopharmacol. 1997;17:370-376. doi: 10.1097/00004714-199710000-00006

\section{EDITORIAL}

1. USPSTF: High blood pressure in children and adolescents: screening. Accessed June 2, 2021. https://uspreventiveservicestaskforce.org/uspstf/recommendation/blood-pressure-in-children-and-adolescents-hypertension-screening

2. Yang L, Magnussen CG, Yang L, et al. Elevated blood pressure in childhood or adolescence and cardiovascular outcomes in adulthood: a systematic review. Hypertension. 2020;75:948-955. doi: 10.1161/hypertensionaha.119.14168

\footnotetext{
3. Falkner B, Lurbe E. The USPSTF call to inaction on blood pressure screening in children and adolescents. Pediatr Nephrol. 2021;36:1327-1329. doi: 10.1007/s00467-021-04926-y

4. Sinaiko AR, Jacobs DR Jr, Woo JG, et al. The International Childhood Cardiovascular Cohort (i3C) consortium outcomes study of childhood cardiovascular risk factors and adult cardiovascula morbidity and mortality: Design and recruitment. Contemp Clin Trials. 2018;69:55-64. doi: 10.1016/j.cct.2018.04.009
} 